\title{
人文地理学的“神经转向”研究进展及启示
}

\author{
王 敏 ${ }^{1}$, 林铭亮 ${ }^{2}$, 朱 痏 $^{3^{*}}$
}

(1. 华南师范大学地理科学学院, 广州 $510631 ; 2$. 华东师范大学城市与区域科学学院, 上海 200241;

3. 广州大学华南人文地理与城市发展研究中心,广州 510006)

\begin{abstract}
摘 要: “神经转向”是在后现代语境下提出的,运用神经机制诠释人的心智与空间的关系, 超越“社会文化”与“生物 神经”的边界, 强调情感、身体、“非理性”等的主体特征, 为人文地理学研究提供了新的认识论和方法论。同时, 人 文地理学研究的 “空间传统” 有益于对批判神经科学关于 “心智一大脑一环境” 的理解产生知识溢出, 实现学科交叉 的良性循环。论文梳理了国内外涉及神经科学理论与方法的人文地理学研究, 基于具身认知和非表征理论分析人 文地理学中 “神经转向” 研究的理论基础, 并围绕空间感知、情感认知、行为实践等主题进行介绍。另外, 神经科学 技术手段为人文地理学研究提供了新的方法, 大脑成像、眼动追踪技术已经在地理学科中得到应用, 呈现可期的未 来愿景。最后,论文提出“神经转向”对中国人文地理学研究的理论创新和实践路径的启示。
\end{abstract}

关 键 词: 神经科学; 非表征理论; 具身认知; 心智;行为; 人文地理学

不同时期的哲学关于 “人是什么”“如何研究 人”的答案往往定义了不同的主体性的理解。随着 近代科学和现代哲学的发展, 人的“心智”(mind)进 人了实验心理学、神经科学、认知科学等自然学科 的研究领域, 它不再是“经验的” “理性的”, 而是“生

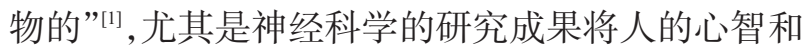
行为与人的神经系统 (主要是大脑)联系起来, 并给 予科学化的解释, 重新定义了人的主体性特征。社 会科学研究的“身体转向”(body turn)使得身体不再 被漠视, 具身心智(embodied mind)认为人的心智与 身体活动不可分离 ${ }^{[2]}$ 。因此, 以身体为核心的主体 性特征受到包括人文地理学在内的社会科学的重 视, 神经活动作为“自然的身体”的重要组成部分, 开始受到人文与社会科学的关注。20世纪 90 年代 起, 神经科学的理论与方法被运用到社会科学研究 之中 ${ }^{[3]}$ 。Callard 等 ${ }^{[4]}$ 所著的《Rethinking Interdisciplinarity across the Social Sciences and Neurosciences》
揭示了神经科学与人文社会科学的交叉研究的重 要性,呼吁社会科学应当关注神经科学在理论和实 践上的影响。

人文地理学的研究核心是“人一地关系”,作为 主体的“人”所反映出的某些特定特征, 对周边的自 然环境或社会环境产生有效的影响是值得我们关 注的 ${ }^{[5]}$ 。“人一地” 关系的互动实质上是由心智支配 的人类活动与环境的互动, 神经科学认为人的心智 应当归结于人的神经机制, 因此促成了人文地理学 中的 “神经转向” (neural turn) ${ }^{[6]}$, 神经活动发生在特 定时空的环境中, 从而产生了人的心智, 神经中枢 能控制人的行为, 对环境作出反作用, 因而神经科 学能为 “人一地关系”的认识论提出新的视角, 从而 为研究人与环境的关系提出新的理解, 引起了人文 地理学家的注意 ${ }^{[7]}$ 。在方法论上, 神经科学视角尝 试超越“自然生物”与 “社会文化”的学科界限, 寻求 学科交叉的研究范式和方法 ${ }^{[8]}$ 。

收稿日期: 2019-05-23; 修订日期: 2019-07-26。

基金项目: 国家自然科学基金项目(41871127,41630635,41601133)。[Foundation: National Natural Science Foundation of China, No. 41871127, 41630635 and 41601133. ]

第一作者简介:王敏(1981一),女,广东汕头人,副教授,博士, 研究方向为社会文化地理与城乡规划。 E-mail: wminmin@m.scnu.edu.cn

*通信作者简介: 朱竑(1968- ), 男, 甘肃临夏人,教授,博士, 博士生导师,研究方向为社会文化地理与旅游地理。 E-mail: zhuhong@gzhu.edu.cn

引用格式: 王敏, 林铭亮, 朱竑. 人文地理学的“神经转向”研究进展及启示 [J]. 地理科学进展, 2020, 39(7): 1182-1195. [Wang Min, Lin Mingliang, Zhu Hong. Research progress and implication of the "neural turn" in human geography. Progress in Geography, 2020, 39(7): 11821195. ] DOI: $10.18306 /$ dlkxjz.2020.07.011 
人文地理学的方法是多元的, 既有定性和定量 方法的争议,也有 “小数据”与 “大数据”的矛盾 ${ }^{\left[{ }^{[]}\right.}$, 而 这些多样化的方法仍置于学科内部的讨论。客观 的“第三人称”(third-person perspective)方法对主观 意识的解释水平往往受到质疑, 缺少了人本主义理 解, 而主观的“第一人称” (first-person perspective)方 法的科学性、准确性亦有待考证, 容易陷人唯心主 义的困境 ${ }^{[10]}$ 。如何使用客观的、科学的方法理解 “第一人称” 视角的世界, 恰是神经科学对人文地理 学最大的启示之一。与此同时, 地理环境深刻地影 响着人们的生活, 神经活动机制受到特定环境的影 响, 地理学对于环境、空间研究的传统, 能够为神经 科学提出理论借鉴。

近 $20 \mathrm{a}$ 来, “神经转向” 在西方人文地理学迅速 发展, 国内地理学、城市规划、旅游等领域亦开始关 注神经科学的视角, 引人神经科学技术的研究已经 出现。但总体而言, 中国人文地理学对神经科学的 影响关注还不足, 本文旨在回顾和梳理神经科学如 何给人文社会科学提供认识论和方法论空间, 阐述 人文地理学是如何接纳神经科学的视角和方法及 其衍生的研究话题, 以期为中国人文地理学的研究 提供借鉴和启示。

\section{1 理论支撑:具身认知与非表征理论}

“神经转向” 是在 “人类是非理性的” 的后现代 语境下提出的, 以人的神经系统为主体, 致力于理 解其结构和功能、如何工作, 以及如何影响行为实 践和获得心智的能力, 强调人的身体、情感、“非理 性” 的主体性地位 ${ }^{[2,11]}$ 。神经科学与人文地理学的跨 学科对话, 需要找到学科间的共通点, 批判神经科 学强调神经机制的空间特征和情境性, 为人文地理 学提供了研究话题的空间; 同时, 具身认知地理学 和非表征理论亦从神经科学寻得理论支撑, 实现学 科间的相互联系、相互发展。

\section{1 批判神经科学: “心智一大脑一环境”}

神经科学起初以本质主义为哲学基础, 即主张 知识的统一性, 其中 “还原论” 认为人的心智和行为 活动都能归因于生理本身, 人的心智能够完全地被 复杂的神经机制解释 ${ }^{[12]}$, 有着将人归因于生物学的 自然主义倾向 ${ }^{[6]}$ 。

随着神经科学的发展, “还原论” 遭到批判, 出 现了批判神经科学(critical neuroscience), 其主张关
注不同情境、空间和身体中的神经机制 ${ }^{[13]}$,认为:第 一, 人的生理现象和心理现象存在联系,但物理现 象无法完全解释心理现象, 不能只将人的身体置于 物质的范畴之中 ${ }^{[14]}$; 第二, 神经科学的知识不只是 对大脑的研究, 要将大脑置于不同的身体, 再将身 体置于不同的环境中理解, 神经科学要转向对“心 智一大脑一环境” (mind-brain-environment) 的庞大 系统的研究 ${ }^{[15]}$, 人的心智并不只在自身发展, 还需 要在主体间的相互作用过程中形成 ${ }^{[16]}$; 第三, 实验 室情境与真实环境存在矛盾 ${ }^{[17]}$, 实验室作为科学家 在特定时间、特点地点所做的特定工作的空间，结 果受到实验情境的影响, 因此实验室以外的多元化 环境可能会产生不同的认识,神经科学研究应存在 “非本质”的知识 ${ }^{[18-19]}$ 。

批判神经科学尝试打破自然和文化的二元界 限, 关注人的身体所处的真实地理环境和神经活动 所处的文化语境 ${ }^{[20]}$, 在技术上重视研发适用于真实 环境的设备, 如可穿戴式设备等 ${ }^{[2]}$, 同时亦关注神 经科学知识对社会的影响 ${ }^{[22]}$ 。因此, 批判神经科学 需要其他学科, 尤其是社会科学的支持 ${ }^{[23]}$ 。神经科 学为人文社会科学提供了新的认识论和方法论,使 得社会科学和神经科学的联系逐渐密切 ${ }^{[8]}$, 从而形 成许多新兴的子学科, 如神经经济学 ${ }^{[24]}$ 、神经营销 学 ${ }^{[25]}$ 、神经教育学 ${ }^{[26]}$ 、神经人类学 ${ }^{[27]} 、$ 神经现象学 ${ }^{[28]} 、$ 神经管理学 ${ }^{[29]}$ 等。批判神经科学关注神经机制所 处的地理环境, 恰是有助于人文地理学对“人一地 关系”的讨论进一步延伸到“心智”与“环境”的关 系, 尤其是对“人一地关系”中非表征、具身的视角 与方法的补充。

\section{2 具身认知与非表征理论: “大脑一身体一环境”}

神经科学将以大脑为核心的神经机制作为解 释的基础,而神经活动与环境相互作用之间是以人 的身体为中介的 ${ }^{[4]}$, 因此“身体转向”所强调具身认 知(embodied cognition)范式及关注身体实践的非表 征理论(non-representational theories)为人文地理学 与神经科学学科交叉搭建了理论桥梁, 而“神经转 向”有助于将抽象化的理论置于具体的实践之中。

随着“身心关系”的转变, 心智的理解从“离身” (disembodied)转向 “具身” (embodied)。笛卡尔的 “身心二元论”认为人是拥有脱离身体之上的纯粹 心智 ${ }^{[30]}$ ，“身体”处于被忽视的地位, 社会科学关注 人们的“心灵”, 而身体则归人生物学研究 ${ }^{[31]}$ 。“二元 论” 将人的意识理解为 “离身心智” (disembodied 
mind), 人的认知等同于运算, 认知过程是对一系列 符号的联结、加工和表征 ${ }^{[32]}$, 据此运用 “心理语法” 去理解人的语言能力 ${ }^{[33]}$, 关注心智、语言和文化的 关系, 将文化理解为运用符号表征的系统, 以建立 一套具有普遍性的认知图式, 即“表征主义” ${ }^{[34]}$ 。从 尼采将身体置于哲学的中心位置开始, “身心二元 论”遭到批判 ${ }^{[35-36]}$, 布迪厄提出 “惯习”以强调身体的 实践 ${ }^{[37]}$, Merleau-Ponty ${ }^{[37]}$ 的知觉现象学提出了“具身 的主体性” (embodied subjectivity) 概念, 认为人以 “体认”的方式认识世界、他人和自己。在“身心一 元” 的语境下, 对人的认知的理解转向具身化, 其认 为身体与心灵不可割裂, 强调身体在认知过程的作 用: (1) 身体的结构和功能决定了心智的内容和属 性; (2) 心智是根植于环境的, 心智、身体和环境融为 一个整体 ${ }^{[39]}$ 。心智的本质是基于身体的, 人类抽象 的思维来自于身体的感觉而非隐喻符号的加工 ${ }^{[40]}$ 。 “身体有自己的记忆, 储藏着各种无意识的知识” ${ }^{[4]]}$, 自 20 世纪 70 年代的 “身体转向” 开始 ${ }^{[2]}$, 人文地理学 转向对物质的关注, 理解地理中人的 “肉体” 是什 么, 将 “具身认知” 置于人文地理学问题中去讨论, 从跨学科的理论中获得启示, 研究涉及身体的空间 性以及生活在空间和地方的情感和行为方面 ${ }^{[22]}$ 。

在具身认知的认识论下, 非表征理论将人的主 体性置于具身情感、身体实践和行为展演中, 并以 神经科学证据作为支撑, 成为了 “神经转向” 的理论 切人点。非表征理论认为人处于 “湍流” (outflows) 式变化的环境中, 只能对小部分的意识有明确认 知, 而其余则处于 “无意识” (unconscious) 状态 ${ }^{[43]}$ 。 非表征理论不仅关注可被认知的意识, 还需要探讨 正在发生的意识的动态轨迹(dynamic trajectory) $)^{[44]}$, 包括了人的微观神经活动和人的 “具身化” 的感觉, “无意识”的过程发生于当下人的身体, 要从身体动 作、行为和具身情感中获得意义 ${ }^{[45]}$, 关注日常生活中 的“表征”(presentations)、“展示”(showings)和“表现” (manifestations), 强调身体的情感反应 ${ }^{[4]}$ 。由于“表征 主义”难以捕捉瞬间的、细微的日常生活变化 ${ }^{[4]}$, 非 表征理论具身化的目标是将语言意义与人的认知 相互分离, 将认知视为基于无意识经验的综合功能 过程 ${ }^{[46]}$ 。Thrift ${ }^{[47]}$ 提出将人的意识要被看作是 “心智 生态” (an ecology of the mind), 即人在不同的环境 中, 心智使身体呈现不同的展演, 这些展演是受到 神经控制的, 研究其背后的神经机制即可了解人的 心智。非表征理论是新文化地理学新趋势, 受到国
内外学者的关注, 并反思人文地理学研究方法的创 新 ${ }^{[8]}$ 。非表征理论的代表学者 Thrift 从神经科学的 研究成果中寻求证据, 如 Libet ${ }^{[49]}$ 的实验发现人的意 识是需要时间建构的, 在意识影响我们的心理活动 之前, 大脑活动会有相当大的延迟, 这一时间空隙 被称为“意识的时间”,即 Thrift 所述的“无意识”的 状态, 亦是神经活动的过程 ${ }^{[4]}$; 人类大脑中某些特 定的神经过程先于特定的感觉, 这些神经过程能够

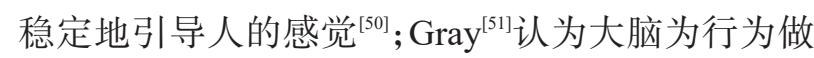
好准备, 从而让人产生了行动的经验。“镜像神经 元”(mirror neurons)的发现也印证了人的身体行为 与神经活动存在着对应关系, 人的心智活动和行为 展演不完全依赖于语义的理解, 而是身体与外界环 境的相互作用 ${ }^{[52]}$ 。

作为自然科学的神经科学并不是与身体的社 会化解释相对立的, 而是从身体的自然属性去诠释 情境性的空间身体感知、具身情感、身体实践与展 演等 ${ }^{[7]}$, 因此人文地理学的具身认知与非表征理论, 能够与批判神经科学相契合, 从而为 “神经转向”的 研究实践提供理论基础。

\section{2 研究主题:感知、情感、实践}

“神经转向” 旨在寻求人的认知及行为与生理 机制之间的关系 ${ }^{[53]}$, 而这一视角的贡献在于能关注 微小的、“无意识”的状态, 有效地回应非表征理论 强调的日常生活实践中不依赖于语义理解的身体 实践、展演和具身情感。在此过程中, 人首先与环 境发生感知觉的过程, 即产生具身的空间感知, 继 而在具身情境下发生情感的处理和认知, 并作出身 体展演和行为轨迹反作用于地理环境。据此, 本文 从“空间感知” “情感认知” “行为认知与实践” 3 个方 面进行梳理阐述。

\section{1 空间感知}

行为是人文地理学的关注对象之一, 尤其是行 为地理学开创了从个体行为角度理解空间特征及 形成机制的理论和方法, 从时间地理学、活动分析 法等角度对人行为所构建的生活空间及其分异进 行研究 ${ }^{[54-56]}$, “神经转向”与行为地理学同样关注人 的主观能动性, 但是 “神经转向”更关注身体尺度上 的行为, 主张将空间中的社会现象与神经机制相联 系, 对人的关注需要回到人的身体乃至大脑 ${ }^{[6]}$ 。因 此, 神经科学的视角更关注人在空间的具身的生理 
感受, 是对行为空间的动机的进一步理解,包括“感 觉” 和 “知觉” 两方面, 感觉是人直接的器官感受, 包 括视觉、嗅觉、听觉等, 知觉是经过一系列信息加工 后得到的结果 ${ }^{\left[{ }^{[7-58]}\right.}$ 。

空间感知主要关注人对实体空间的位置、方 向、几何特征、颜色、景观特征等的识别。Sholl ${ }^{[59]}$ 从 实验心理学、神经科学的视角解释成年人的空间定 位问题, 认为步行行为受到视觉神经系统的控制, 而目标导向受到大脑系统的控制; Mealey 等 ${ }^{[60]}$ 通过 视觉实验发现当人首要识别并习惯于看某一定向 的地图时, 其他定向地图会延长人的视觉对信息的 处理时间, 甚至产生 “定向错误”; Zeise $\mathrm{e}^{[6]]}$ 的研究发 现阿尔茨海默症的患者损伤的海马体负责处理空 间定位, 疗养场所的空间设计会影响患者的恢复 速度; Hillier ${ }^{[62]}$ 发现人对于城市的认知是基于几何 直觉的空间结构规律, 是人的思维与城市环境相互 作用的基础; Tang 等 ${ }^{[63]}$ 发现城市的水体环境有助于 降低人的注意力水平; Dadvand 等 ${ }^{[6]}$ 发现长期生活 在绿化环境的儿童比同龄人有更高的认知水平等; 王敏等 ${ }^{[65}$ 从眼球运动特征研究居民对城市开敞空 间各要素的感知特征, 以对公共空间规划提出生理 指标的意见; 龙瀛等 ${ }^{[6]}$ 基于图片城市主义的理论和 方法, 运用眼球运动数据提出城市街道品质的评 价方法等。

这类研究有明确的目标和应用性, 直接将人的 生理反应与实体空间特征联系, 尤其是与日常生 活、健康、身心发育等问题密切相关, 有助于在环境 设计和城市规划中, 提供量化的统计分析数据作为 支持,有效克服研究者主观因素的干扰 ${ }^{[6]}$ 。

\section{2 情感认知}

情感对空间与社会发展产生影响, 情感地理成 为人文地理学所关注的重要话题之一 ${ }^{[68]}$, 情感认知 是人内隐的心理状态, 蕴藏于空间中的情感因素往 往难以用言语表征 ${ }^{[6]}$, 人与地方的情感联系是主观 而抽象的。在研究视角上, 神经科学提供了 2 种路 径:一种是从情感神经科学(affective neuroscience)中 寻求解释, 用生理激素和神经生物学的机制解释情 感的产生 ${ }^{[70]}$; 另一种则从外部出发, 挖掘文本、图像、 景观等表征符码背后的神经因素, 如Lakoff 等 ${ }^{[71-72]}$ 提 出的是认知语言学的方法, 他所描述的认知框架即 人的思维结构, 是一种根深蒂固的、处于无意识层 面的结构, 在生活中长期重复使用而变得难以改 变, 这一套固定的框架环路(frame-circuitry)植根于
人的大脑之中, 体现在人的神经突触上, 以神经回 路的形式存在于身体中; Connolly 则强调身体和仪 式的实践, 情感是在实践的相互作用之中形成的, 情感存在于共鸣的不同身体或景观之中 ${ }^{[73]}$ 。

在 “情感转向”下, 人文地理学所涉及的话题十 分丰富。在情感的作用下, 人赋予空间特定意义而 形成地方,地方性研究的情感因素是神经科学所关 注的对象之一。Lengen 等 ${ }^{[74]}$ 运用神经科学证据解 释地方认同(place identity)的产生及其作用,认为人 在地方的长期生活必然产生与地方相关的 “神经 元” 特征, 形成特殊的情感联系; Pykett 等 ${ }^{[75]}$ 从文化 地理学和神经科学的角度, 分析英国人力资源领域 的培训项目及其基于积极心理学设计的工作场所, 在特定的情境空间中形成“大脑文化” 以及心理治 理的模式对于强化工作场所绩效有密切联系; 王敏 等 ${ }^{\left[{ }^{[6]}\right.}$ 通过追踪被试的眼球运动发现本土节庆的参 与者与外界 “他者”对节庆的身体展演空间的凝视 中瞳孔放缩反应有着显著的差异, 地方的情感因素 在人观看的过程中发挥着作用; Montag 等 ${ }^{\left[{ }^{[7]}\right.}$ 从大脑 情感的视角分析了数字媒体技术对人产生的影响, 互联网带来的实体与虚拟的空间交互在重塑人类 的大脑, 改变人们对地方的认知。

旅游地理的研究集中于旅游者行为的动机及 其在旅游体验。Walls 等 ${ }^{[78]}$ 从 Naqvi 等 ${ }^{[79]}$ 的神经科学 研究成果证明大脑存在 2 个或 2 个以上不同的决策 处理区域, 认知和情感作为决策系统中重要部分, 独立地发挥着作用, 呼吁关注情感在旅游决策行为 过程中的影响及其神经机制; Pearce ${ }^{[80]}$ 从神经科学 视角整合人的情感与记忆、时间感知等因素, 研究 人 “回到熟悉的地方” 的旅游主题; Goggin 等 ${ }^{[81]}$ 通过 对游客具身化的情感测量, 分析游客与原住民是如 何与环境发生身体感官、认知和情感联系的。

在政治地理的话题上,主要是情感地缘政治 (emotional geopolitics)的研究, 其认为情感在国家、 民族和地缘战争等方面有着重要作用, 是重构地缘 政治力量和文化意义的重要因素 ${ }^{[82]}$, 要将日常生活 的情感与政治的过程联系起来, 以解释人类行为背 后的机制 ${ }^{[83]}$ 。Ivakhiv ${ }^{[84]}$ 发现人的无意识层面是政治 的情感沟通,认为环境保护主义的宣扬上要塑造多 元化的框架,并更多地关注语言和实践对行动、交 流和居住的社区的情感影响; Barr 等 ${ }^{[85}$ 探讨了各国 应对气候变化问题的措施,认为政策制定者要从个 体的神经行为特征出发提出有效的解决方法; Ga- 
$\operatorname{gen}^{[86]}$ 研究近年英国的学校将情感纳人公民教育之 中, 将学生的情感技能进行标准化测定, 运用情感 神经机制强化公民身份, 通过学校空间重塑其身份 认同; Bille ${ }^{[8]}$ 援引神经科学的躯体失忆症的观点提 出了“民族即身体”的论述, 国家领土的失去如同身 体截肢的痛苦, 失去的领土依然会存在于民族情感 和想象之中, 形成“幻痛”的记忆。

人文地理学逐步重视情感的作用, 尤其在社会 文化空间的研究之中, 情感对空间的重塑和建构具 有深刻意义。神经科学的方法有助于运用客观的 表征方式更好地理解人的情感, 丰富情感地理理 论, 挖掘“人一地关系”中的社会文化逻辑。

\section{3 行为认知与实践}

随着神经科学知识的普及, Pykett ${ }^{[88]}$ 认为神经 科学已经渗透于城市公共政策的制定之中, 推动政 策实验项目以研究公民的 “大脑文化” (brain culture), 即大脑的知识、图像及其表征运用于文化身 份和社会认同的重塑中, 影响公民的情感和行为实 践, 这种政策主张被称为“神经自由主义”(neuroliberalism); $\operatorname{Isin}^{[89]}$ 首先提出了神经自由主义的概念, 其 将“神经性质的公民”(neurotic citizen)作为主体, 通 过神经机制运用人的认知策略、情绪和先于认知的 情感确保公民首选某一种社会行为的治理方式, 即 利用人的神经活动特征来管理人的行为, 其最终目 的是重塑公民的行为实践, 是一种非话语的权力运 作 ${ }^{[00]}$, 主要体现在西方人文地理学的批判性研究 中,尤其是经济和政治 2 个领域。

在经济领域, 随着古典经济学中的 “经济人假 设”逐渐弱化, 人的“有限理性”的假设开始流行 ${ }^{[91-93]}$, 行为经济学应运而生。神经科学认为人的经济决 策是基于大脑无休止的冲突产生的, 神经系统对个 人的动机、注意力、认知等方面的控制深刻地影响 着人的决策。神经经济学将人的大脑作为经济主 体, 研究人的大脑如何做出经济决策, 是一种将经 济理论与神经科学实证方法结合起来的学科 ${ }^{[4-95]}$ 。 经济地理学认为人的行为不仅是个体的、心理的, 还与环境有关, 经济地理学能够提供一个研究人的 经济行为与环境相互作用的研究视角 ${ }^{[96]}$, 神经科学 则为这一研究视角提供神经生理的解释。神经经 济学为理解人的行为提供新的角度, 已经渗透到了 商业应用、公共政策、市场营销等多个方面 ${ }^{[24]}$, 如 Lewis ${ }^{[97]}$ 结合卡尼曼风险定律的实验分析认为人的 偏好与市场环境变化存在着密切联系, 市场环境的
波动乃至金融危机与人的 “短视”行为有关, 而神经 科学证据表明这种行为在特定的时空间发生, 是人 类的一种根深蒂固的生物学特征; Tanja 等 ${ }^{[25]}$ 运用脑 成像的神经科学技术了解消费者是如何感知市场 产品的, 将神经科学运用到消费之中; Proffitt ${ }^{[98]}$ 运用 人对空间布局的视觉认知与执行预期行为的成本 费用的关系理解人们经济行为的差异。

在政治领域上学者开始关注渗透于公共政策 中的与神经科学有关的内容,如英国政府于 2010年 成立行为观察小组, 2013 年成立 Nudge Unit, 倡导 使用行为科学、心理学、神经科学的知识制定政策; 法国于 2010 年出台与神经科学有关的政策, 报道 了神经科学的公共卫生影响及其法律含义 ${ }^{[99]}$; 丹 麦成立了一个非盈利组织iNUdgeYou, 主要为公共 政策和商业公司提出行为科学的设计; 新加坡的人 力资源部成立了行为观察与设计部门; 美国白宫已 经建立了社会和行为科学项目 Nudge Squad ${ }^{[88]}$ 。另 一方面,在政策制定、可持续发展议程等方面开展 了实证研究, 如 $\mathrm{Grasso}^{[100]}$ 认为道德认知神经科学的 双过程理论有助于为基于结果主义方法的气候道 德认知判断提供依据, 有利于解决人类在面对涉及 非个体伤害概念的气候变化等问题时的道德判断 问题; Yalachkov 等 ${ }^{[101]}$ 从神经科学的证据发现人们 对汽车的依赖是长期形成的源于城市日常工作和 学习压力的行为习惯, 所以可替代的绿色出行模式 是难以推进的, 需要结合地理学和城市规划的相关 研究理解人们是如何与日常环境进行互动, 并提出 有效政策; Barr 等 ${ }^{[85}$ 探讨了在各国为解决全球气候 变化的努力中不断审视个人角色的地位,通过改变 物质环境和消费环境, 继而改变个体行为从而推动 可持续发展的交通政策。

神经科学为社会现象的批判性理解提供了路 径, 能更好地剖析社会空间中的正义、道德和权力 运作等问题, 需要关注行为情境的空间特征(the spatial qualities of behavioural context) 以及公共空 间与政策是如何重塑情境的 ${ }^{[102]}$ 。神经科学的知识 同时能作为 “非理性” 的话语带有规范性价值的假 设,将公民行为导向健康的、理性的、可持续的社会 生活 ${ }^{[103]}$ 。

\section{3 神经科学技术手段}

神经科学的研究基础为人文地理现象提出了 
许多可供参考的证据，同时提供新的方法和技术。 神经科学的方法主要分为侵人性(invasive) 和非侵 人性(non-invasive)方法, 侵人性的技术主要运用于 动物、脑损伤的病人 ${ }^{[104]}$,一般不能用于正常的被试, 应用范围相对狭窄。所以本文主要介绍非侵人性 的相关方法, 这些实验方法一般不会对被试产生有 害影响, 主要是采集生理数据, 运用实验仪器捕捉 人体微小的生理变化, 并转化为数字、图像等可视 化数据(表 1$)$ 。

\section{1 大脑成像}

人在接收认知事件刺激时, 会导致大脑的物质 新陈代谢发生变化, 而不同的刺激物会引起不同脑 区的变化, 大脑成像是利用该原理,将人的大脑的 物质变化转变为二维或三维的可视化图像 ${ }^{[107]}$ 。磁 共振成像(MRI)、功能性磁共振成像(fMRI) 是目前 较为常用的脑成像技术, 磁共振成像的优势在于可 以发现整个大脑的神经活动, 并在图像上以体素 (voxel)进行定量化描述 ${ }^{[108]}$ 。

大脑成像技术在地理学中有初步应用, 如 Lederbogen 等 ${ }^{[109]}$ 使用 fMRI 技术研究城市精神疾病与 城市环境的关系, 发现了生活在不同教养(upbringing)的城市中人们的压力情况具有显著差异, 原因 主要在大脑中杏仁核活动和扣带皮层对压力的调 节; Tang 等 ${ }^{[63]}$ 使用 fMRI 研究城市设计中自然环境 与人的有意识注意水平的关系, 发现森林景观与城 市景观在大脑注意力恢复水平上没有显著差异, 山 脉和水环境有显著差异, 其中水环境还引起了大脑 的背后扣带皮层 BA31 区发生反应; Dadvand 等 $^{[64]}$ 用 MRI 技术研究了长期生活于绿化环境中的儿童的 工作记忆和注意力水平的关系, 该研究以白质和灰
质为主要指标, 发现生活在不同绿化环境的儿童脑 区存在显著差异,这些有差异的脑区与处理注意力 和工作记忆的脑区部分重合。

fMRI 和 MRI 技术对实验环境的要求较高, 仪 器的复杂性、实验环境的封闭性、对被试的高要求 等都成为了大脑成像应用的限制性因素,但近年来 出现的新技术, 如功能性近红外光谱技术(fNIRS)具 有便携性好、造价较低的优势, 能应用于真实环境 中 $^{[05]}$, 有较强的应用潜力。可穿戴的神经实验设备 是目前神经科学技术研发的重要方向, 为神经科学 方法应用于真实环境提供了可期的愿景 ${ }^{[2]}$ 。

\section{2 眼动追踪技术(eye tracking)}

人文地理学“文化转向”背景下,视觉文化受到 了地理学的关注, 并从研究视觉材料表征的意象转 向视觉材料的建构与观看的非表征过程,更注重 “看的方式” ${ }^{[110]}$ 。而视知觉(visual perception)是意识 的类型之一, 主要指视觉上的主观体验, 是研究人 的意识的重要切口, 原因在于视觉有着丰富和特别 的信息,可以更好地揭示意识的主观特征,并且视 觉研究更容易进行操作性定义 ${ }^{[111]}$ 。研究视知觉的 神经科学技术主要是眼动追踪技术, 目前常用的工 具是眼动仪。眼动仪主要是捕捉被试的眼动活动, 包括了瞳孔反应、注视点、注视时间等。眼动能够 反映被试的情绪和认知的动态性变化, 同时眼动作 为一种自主的生理数据, 能够更加真实地反映被试 在实验过程中的状态。眼动实验具备容易操作、轻 巧灵活的特征,使得实验更具有可行性 ${ }^{[106]}$ 。

眼动仪在国内外地图阅读、GIS 系统设计、旅 游、城市规划等方面都有较多的应用。董卫华等 ${ }^{[12]}$ 详细介绍了眼动仪 2008 年以来的应用研究进展, 认

表 1 神经科学主要的非侵入性方法及其应用展望

Tab.1 Major non-invasive approach in neuroscience and prospect of applications

\begin{tabular}{|c|c|c|c|c|}
\hline 观测对象 & 方法名称 & 原理 & 数据产物 & 应用 \\
\hline \multirow[t]{2}{*}{ 大脑 } & $\begin{array}{l}\text { 计算机断层扫描(CT/CAT)、磁共振脑成 } \\
\text { 像(MRI)、功能性脑成像(fMRI)、功能性 } \\
\text { 近红外光谱技术(fNIRS) }\end{array}$ & $\begin{array}{l}\text { 利用大脑在认知事件刺激下发生的 } \\
\text { 磁性、电流或新陈代谢变化进行测定 }\end{array}$ & 大脑图像 & $\begin{array}{l}\text { 观测特定脑区的变化,从 } \\
\text { 而判断环境刺激与对应大 } \\
\text { 脑功能的关系 }\end{array}$ \\
\hline & 脑电图(EEG)、事件相关电位(ERP) & 记录神经突触活动产生的电流 & $\begin{array}{l}\text { 脑电变化过程 } \\
\text { 数据 }\end{array}$ & $\begin{array}{l}\text { 观测大脑处理认知事件的 } \\
\text { 应激变化 }\end{array}$ \\
\hline 皮肤 & 皮肤电活动(EDA) & $\begin{array}{l}\text { 人的皮肤微弱的电流活动受到认知、 } \\
\text { 情绪变化的影响而变化 }\end{array}$ & $\begin{array}{l}\text { 皮肤电变化过 } \\
\text { 程数据 }\end{array}$ & 观测情绪、认知变化过程 \\
\hline 心脏 & 心率(HR)、心率变异系数(HRV) & $\begin{array}{l}\text { 心脏的舒缩会引起动脉血管内的压 } \\
\text { 力变化,从而导致管壁发生搏动 }\end{array}$ & 心率变化数据 & 观测情绪变化过程 \\
\hline 眼球 & 眼动仪(Eye-tracking) & 捕捉人眼球反射的红外线 & $\begin{array}{l}\text { 视觉指标、瞳孔 } \\
\text { 数据、注视点轨 } \\
\text { 迹和热点图 }\end{array}$ & $\begin{array}{l}\text { 观测眼球运动过程, 推导 } \\
\text { 认知和情感的变化 }\end{array}$ \\
\hline
\end{tabular}

注:本表根据文献[17,104-106]整理。 
为眼动追踪方法对于理解人们的地图认知十分重 要, 眼动参数是地图制图的重要参考 ${ }^{[113-114]}$ 。另外, Wang 等 ${ }^{[115]}$ 研究被试对旅游照片的眼动反应, 挖掘 旅游地在视觉上的吸引因素; 王敏等 ${ }^{[116-117]}$ 将眼动仪 运用于文化遗产的研究中, 发现眼动轨迹的热点区 域与人们对景观符号的记忆情感有关, 认为眼动研 究是保护文化遗产原真性的有效路径; Bodor ${ }^{[118]}$ 运 用眼动仪发现城市居民与乡村居民在对环境探索 的能力上存在差异; 龙瀛等 ${ }^{[66]}$ 将眼动实验用于街道 品质的评价之中。

眼动追踪技术能够有效地提供捕捉人的观看 过程中生理数据变化的方法, 传统上被试是在眼动 实验室中观看图像或视频, 与真实环境存在一定误 差。而随着技术的深化发展, 眼动仪能够安装到智 能眼镜上, 被试能够在真实环境中完成眼动实验 ${ }^{[119]}$, 同时虚拟现实技术(virtual-reality technology) 为眼 动追踪提供了新的环境建构方法, 尤其是对行动不 便特殊人群有更加广泛的应用 ${ }^{[120]}$ 。

\section{4 启示与展望}

本文梳理了人文地理学 “神经转向” 的进展文 献, 发现 “神经转向” 为理解心智作为人的主体性特 征提供了新的视角和方法, 用神经机制解释人的心 智和行为。批判神经科学强调大脑嵌于身体, 身体 嵌人真实环境的理解, 将实验室情境转变为真实世 界的情境。非表征理论认为日常生活中人的心智 大多处于 “无意识” 层面, 并得到神经科学成果支 撑, 要求在具身的情感体验与实践中获得意义。“神 经转向” 既是理论研究的新视角, 也成为理解人与 环境关系的新路径。同时, 神经科学所使用的技术 成为人文地理学新的研究方法, 大脑成像、眼动追 踪技术已经在地理学中得到应用, 并展露出可期的 未来愿景。

“神经转向” 涵盖了空间感知、情感认知、行为 认知与实践的相关话题, 在不同尺度下的地理环境 研究都有可参考的价值(图 1)。神经科学的视角是 面向人内隐的心理结构和生理感受, 对于中国新型 城镇化建设、城市规划、城市更新、社区治理、空间 设计、生产消费、旅游规划等实践应用都有着良好 的参考价值。在当今中国城市化的进程中, 生活空 间质量受到重视，但目前城市中出现的环境问题、 健康问题、城市更新、空间正义、“非正规”的就业与

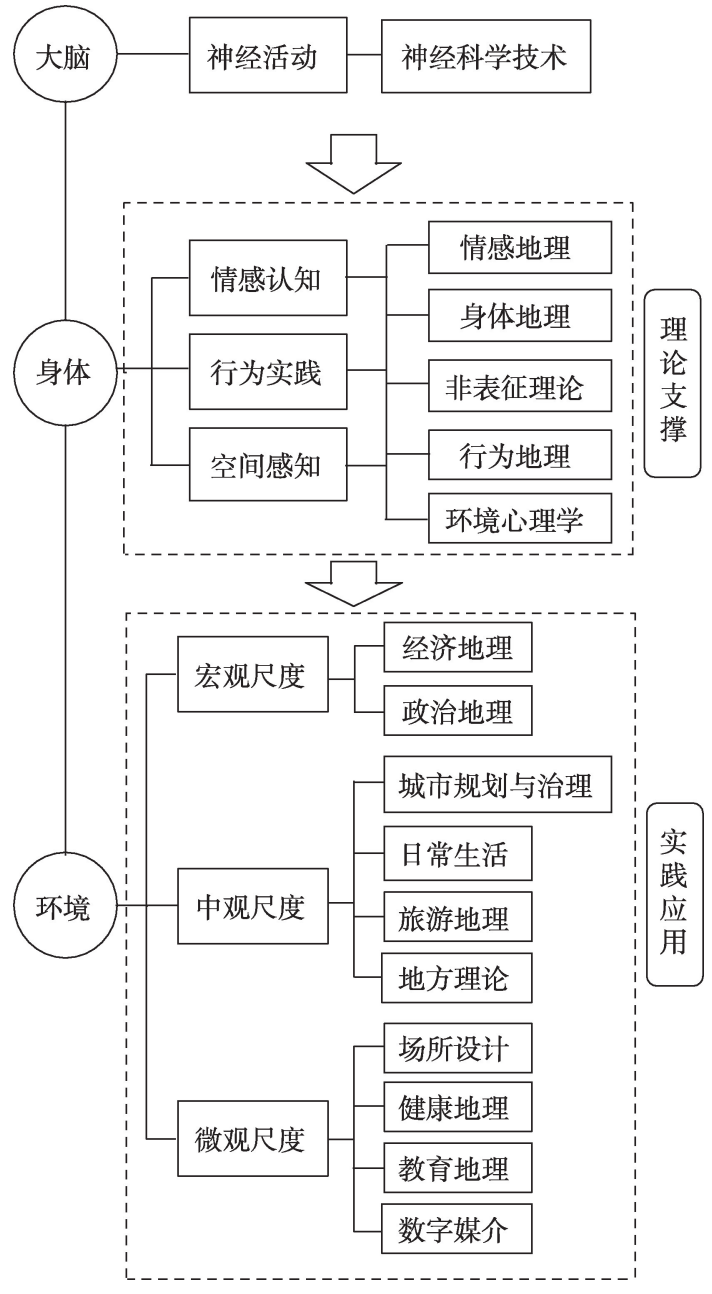

图 1 人文地理学“神经转向”研究框架

Fig.1 Framework of the "neural turn" in human geography 生活空间等关系到“以人为本”的城市建设的落实, 这一过程不仅仅关注人们外显的态度和话语表达， 更重要的是其身体上的“真实感受”,通过地理环境 唤醒积极情感, 实现身体和心理上的良好发展, 都 是“神经转向”在当今中国城市建设中的重要价值。

从人文地理学学科发展上看, 神经活动的情境 性和空间特征是人文地理学应有所为的重要方向, 尤其强调非理性的感知、情感在日常行为中发挥的 作用, “神经转向”不仅意味着人文地理学对神经科 学技术手段的引人,也是对“人一地关系”的新型理 解, 重新认识人的主体是什么, 人的意识是什么。 由此,本文提出以下对未来研究的启示:

(1) 第一人称与第三人称视角的结合成为一种 新的研究范式。神经科学既为社会科学提供了新 的认识论和方法论空间,但也需要社会科学提供理 论支持以接近更加真实的 “真相”, 仅仅从神经科学 技术得到的生理指标并不能揭示深层次的文化意 
义理解 ${ }^{[121]}$, 因而神经科学的实验结果无法反映本质 的世界, 它如同 “实践的美学”让社会科学得以加人 实验性的原则 ${ }^{[122]}$, 如神经人类学、神经现象学的方 法, 提供了第一人称与第三人称方法相结合的视 角。人的心智和行为发生在复杂的真实环境之中, 实验性原则并不完全适用于真实情境, 研究中既强 调对现象的观察以及研究对象的主观的语言表述, 又引人神经科学的实验证据, 以挖掘第一人称视角 与第三人称视角对问题的解释水平 ${ }^{[10]}$ 。

(2) 充分理解神经科学的原理和技术方法。尽 管近年来学术界呼吁社会科学要关注神经科学的 理论成果, 但学科的边界成为了二者难以逾越的鸿 沟, 引入神经科学技术在实践操作层面上是相对困 难的 ${ }^{[123]}$ 。一方面, 神经科学技术所使用的仪器专业 化水平高, 价格昂贵, 操作繁琐, 要进行大样本的研 究时需要花费大量的金钱和时间成本; 另一方面, 人文地理学更加关注一些显性的、顺向推理的问 题, 而神经科学的实验往往是逆向的假设与验证, 因而在神经科学方法运用过程中需要转变思维方 式, 同时在实验过程中提供什么样的刺激、如何控 制、如何深度解读所得结果等均需要深厚的神经科 学理论基础 ${ }^{[24]}$, 在地理学 “神经转向” 的研究中可能 需要进行广泛的跨学科合作。

(3) 挖掘本土化话题, 发现中国国情与语境背 景下的神经科学问题。地理学在神经转向背景的 重要贡献是对心智与行为活动的情境性和空间特 征的关注, “心智一大脑一环境”系统因地而异, 因 而立足于中国语境将有利于提出本土化的补充和 创新, 也能更好地将学科发展和国家社会服务结 合起来。

(4) 坚持 “学科本位”, 强调知识溢出。批判神 经科学关注不同情境、空间和身体中的神经机制, 而空间特征和区域分异是人文地理学的研究传 统。神经科学与人文地理学的交叉研究不仅是神 经科学技术为人文地理学提供新的视角和方法, 人 文地理学的 “空间” 传统能够为批判神经科学提出 知识溢出, 正如神经科学为非表征理论提供了科学 化证据, 相信其也能为其他人文地理学的思想提出 验证和补充, 这有助于加强学科间的对话, 提升人 文地理学在其他领域的学科价值。

诚然, “神经转向” 提供了一个新的研究视角, 但目前神经科学在人文地理学中研究应用还存在 困境, 主要体现于神经科学知识复杂, 技术难度较
大, 需要较多的人力和资金支持,也有赖于与神经 科学学者的合作研究; 神经科学在人文地理学研究 中仍处于探索和尝试阶段,在理论建构上还缺少大 量而深度的实证支撑。但 “神经转向”的思考是值 得借鉴的, 它不仅有助于对人文地理现象和机制提 出更深刻和科学的解释, 也能够为 “人一地关系”提 出创新性的理解。本文仅是对目前“神经转向”的 研究进展进行梳理并提出粗浅的看法, 神经科学如 何与实证研究碰撞出更多的火花,需要人文地理学 学者进一步探索和挖掘, 也需要更多学者的共同参 与和努力。

\section{参考文献(References)}

[1] Gazzaniga M, Ivry R, Mangun G, et al. Cognitive neuroscience: The biology of the mind [M]. Cambridge, USA: MIT Press, 1998.

[2] 陶伟, 王绍续, 朱竑. 身体、身体观以及人文地理学对身 体的研究 [J]. 地理研究, 2015, 34(6): 1173-1187. [Tao Wei, Wang Shaoxu, Zhu Hong. The body, the view of body, and the study of body in human geography. Geographical Research, 2015, 34(6): 1173-1187. ]

[3] Rose N, Abirached J M. Neuro: The new brain sciences and the management of the mind [M]. Princeton, USA: Princeton University Press, 2013.

[4] Callard F, Fitzgerald D. Rethinking interdisciplinarity across the social sciences and neurosciences [M]. London, UK: Palgrave Macmillan, 2015.

[5] Ernste H. The pragmatism of life in poststructuralist times [J]. Environment and Planning A, 2004, 36: 437-450.

[6] Korf B. A neural turn? On the ontology of the geographical subject [J]. Environment and Planning A, 2008, 40(3): 715-732.

[7] Pykett J. Geography and neuroscience: Critical engagements with geography's 'neural turn' [J]. Transactions of the Institute of British Geographers, 2017, 43(2): 154-169.

[8] Fitzgerald D, Callard F. Social science and neuroscience beyond interdisciplinarity: Experimental entanglements [J]. Theory Culture \& Society, 2015, 32(1): 3-32.

[9] 王丰龙, 刘云刚. 多样 vs. 分裂: 论中国人文地理学发展 的多元性及其挑战 [J]. 地理科学, 2019, 39(1): 22-30. [Wang Fenglong, Liu Yungang. Diversity vs. division: The pluralism and its challenges for contemporary Chinese human geography. Scientia Geographica Sinica, 2019, 39(1): 22-30. ]

[10] Kingstone A, Smilek D, Eastwood J D. Cognitive etholo- 
gy: A new approach for studying human cognition [J]. British Journal of Psychology, 2011, 99(3): 317-340.

[11] Murdoch J. Humanising posthumanism [J]. Environment and Planning A, 2004, 36(8): 1356-1359.

[12] 曾向阳. 意识科学中的研究纲领及其方法论探析 [J]. 广东社会科学, 2010(6): 49. [Zeng Xiangyang. Exploring the research program and its methodology in science of consciousness. Social Sciences in Guangdong, 2010 (6): 49. ]

[13] Choudhury S, Slaby J. Critical neuroscience: A handbook of the social and cultural contexts of neuroscience $[\mathrm{M}]$. New York, USA: Wiley-Blackwell, 2012.

[14] 蒋柯. 认知神经科学还原论预设的困境与可能的出路 [J]. 苏州大学学报(教育科学版), 2017, 5(2): 78-85. [Jiang Ke. The predicament of and possible solutions to reductionism presupposition in cognitive neuroscience. Journal of Soochow Unversity (Educational Science Edition), 2017, 5(2): 78-85. ]

[15] Wheeler M. Reconstructing the cognitive world: The next step [M]. Cambridge, USA: MIT Press, 2005.

[16] Slaby J, Gallagher S. Critical neuroscience and socially extended minds [J]. Theory, Culture \& Society, 2015, 32 (1): 33-59.

[17] Mcdowell K, Lin C T, Oie K S, et al. Real-world neuroimaging technologies [J]. IEEE Access, 2013, 1(1): 131149.

[18] Choudhury S, Nagel S K, Slaby J. Critical neuroscience: Linking neuroscience and society through critical practice [J]. BioSocieties, 2009, 4(1): 61-77.

[19] Davies G. Captivating behaviour: Mouse models, experimental genetics and reductionist returns in the neurosciences [J]. Sociological Review, 2010, 58(S1): 53-72.

[20] Seligman R, Kirmayer L J. Dissociative experience and cultural neuroscience: Narrative, metaphor and mechanism [J]. Culture Medicine \& Psychiatry, 2008, 32(1): 3164.

[21] Picard R W, Healey J. Affective wearables [J]. Personal Technologies, 1997, 1(4): 231-240.

[22] Honneth A. Pathologien der vernunft [M]. Frankfurt, Germany: Suhrkamp, 2007.

[23] Callard F, Margulies D S. The subject at rest: Novel conceptualizations of self and brain from cognitive neuroscience's study of the 'resting state' [J]. Subjectivity, 2011, 4 (3): $227-257$.

[24] Pykett J. Neurocapitalism and the new neuros: Using neu- roeconomics, behavioural economics and picoeconomics for public policy $[\mathrm{J}]$. Journal of Economic Geography, 2013, 13(5): 845-869

[25] Tanja S, Steve W. Technologies of ironic revelation: Enacting consumers in neuromarkets $[\mathrm{J}]$. Consumption Markets \& Culture, 2012, 15(2): 169-189.

[26] Ansari D, De Smedt B, Grabner R H. Neuroeducation: A critical overview of an emerging field $[\mathrm{J}]$. Neuroethics, 2012, 5(2): 105-117.

[27] Domínguez Duque J F, Turner R, Lewis E D, et al. Neuroanthropology: A humanistic science for the study of the culture- brain nexus [J]. Social Cognitive and Affective Neuroscience, 2009, 5(2-3): 138-147.

[28] Varela F J. Neurophenomenology: A methodological remedy for the hard problem [J]. Journal of Consciousness Studies, 1996, 3(4): 330-349.

[29] 朱琪, 陈乐优. 神经经济学和神经管理学的前沿 [J]. 经 济学家, 2007(4): 26-30. [Zhu Qi, Chen Leyou. The frontier and development of neuroeconomics and neuromanagement. Economist, 2007(4): 26-30. ]

[30] Bourdieu P. Pascalian meditation [M]. Palo Alto, USA: Stanford University Press, 2000.

[31] Shilling C. The body and social theory [M]. London, UK: Sage, 2012

[32] 李其维. “认知革命”与 “第二代认知科学”刍议 $[\mathrm{J}]$. 心 理学报, 2008, 40(12): 1306-1327. [Li Qiwei. Cognitive revolution and second-generation cognitive science. Acta Psychologica sinaca, 2008, 40(12): 1306-1327. ]

[33] 袁艈林. 认知科学背景上的语言研究 [J]. 当代语言学, 1996(2): 1-12. [Yuan Yulin. Language studies in the context of cognitive science. Contemporary Linguistics, 1996 (2): 1-12. ]

[34] 韩忠太, 张秀芬. 学科互动: 心理学与文化人类学 [J]. 云南社会科学, 2002(3): 60-65. [Han Zhongtai, Zhang Xiufen. Subject interactivity: Psychology and cultural anthropology. Social Sciences in Yunnan, 2002(3): 60-65. ]

[35] 汪民安, 陈永国. 身体转向 [J]. 外国文学, 2004, 24(1): 36-44. [Wang Minan, Chen Yongguo. Body turn. Foreign Literature, 2004, 24(1): 36-44. ]

[36] 叶浩生. 有关具身认知思潮的理论心理学思考 [J]. 心 理学报, 2011, 43(5): 589-598. [Ye Haosheng. Embodied cognition: A consideration from theoretical psychology. Acta Psychologica Sinica, 2011, 43(5): 589-598. ]

[37] 文军. 西方社会学理论: 经典传统与当代转向 [M]. 上 海: 上海人民出版社, 2006. [Wen Jun. Western sociolog- 
ical theory: Classical tradition and contemporary turn. Shanghai, China: Shanghai People's Publishing House, 2006. ]

[38] Merleau- Ponty M. Phénoménologie de la perception [M]. Paris, France: Éditions Gallimard, 1945.

[39] 叶浩生. “具身”涵义的理论辨析 [J]. 心理学报, 2014, 46(7): 1032-1042. [Ye Haosheng. Theoretical analysis of the meaning of embodiment. Acta Psychologica Sinica, 2014, 46(7): 1032-1042. ]

[40] Lakoff G, Johnson M. Metaphors we live by [M]. Chicago, USA: University of Chicago Press, 2008.

[41] Lawrence R L. Coming full circle: Reclaiming the body [J]. New Directions for Adult and Continuing Education, 2012, 134: 71-78.

[42] Longhurst R. (Dis)embodied geographies [J]. Progress in Human Geography, 1997, 21(4): 486-501.

[43] Vannini P. Non-representational methodologies: Re-envisioning research [M]. Abingdon, USA: Routledge, 2015.

[44] Thrift N. Intensities of feeling: Towards a spatial politics of affect [J]. Geografiska Annaler: Series B, Human Geography, 2004, 86(1): 57-78.

[45] Lorimer H. Cultural geography: The busyness of being 'more-than-representational' [J]. Progress in Human Geography, 2005, 29(1): 83-94.

[46] Butcher S. Embodied cognitive geographies [J]. Progress in Human Geography, 2012, 36(1): 90-110.

[47] Thrift N. Non-representational theory: Space, politics, affect [M]. Abingdon, USA: Routledge, 2008.

[48] 王敏, 江荣影, 朱竑. 新文化地理学中的非表征与再物 质化研究进展 [J]. 地理科学进展, 2019, 38(2): 153163. [Wang Min, Jiang Ronghao, Zhu Hong. "Non-representational", "re-materializing" and the research methods of new cultural geography. Progress in Geography, 2019, 38(2): 153-163. ]

[49] Libet B. Mind time: The temporal factor in consciousness [M]. Cambridge, USA: Harvard University Press, 2004.

[50] Roth G. Worüber dürfen Hirnforscher reden- und in welcher Weise? [J]. Deutsche Zeitschrift für Philosophie, 2004, 52(2): 223-234.

[51] Gray J. Straw dogs: Thoughts on humans and other animals [M]. London, UK: Granta Books, 2003.

[52] Corballis M C. Mirror neurons and the evolution of language [J]. Brain \& Language, 2010, 112(1): 25-35.

[53] Uchino B N. Foundations in social neuroscience [M].
Cambridge, USA: MIT Press, 2002.

[54] 柴彦威, 塔娜. 中国行为地理学研究近期进展 [J]. 干旱 区地理, 2011, 34(1): 1-11. [Chai Yanwei, Ta Na. Recent progress of behavioral geographic research in China. Arid Land Geography, 2011, 34(1): 1-11. ]

[55] 柴彦威, 颜亚宁, 冈本耕平. 西方行为地理学的研究历 程及最新进展 [J]. 人文地理, 2008, 23(6): 1-6. [Chai Yanwei, Yan Yaning, Okamoto Kohei. Development of Behavioral geographic research in Western Countries and its recent progress. Human Geography, 2008, 23(6): 1-6. ]

[56] 申悦, 柴彦威. 基于日常活动空间的社会空间分异研究 进展 [J]. 地理科学进展, 2018, 37(6): 853-862. [Shen Yue, Chai Yanwei. Progress of research on sociospatial differentiation based on daily activity space of urban residents. Progress in Geography, 2018, 37(6): 853-862. ]

[57] 胡正凡, 林玉莲. 环境心理学 [M]. 北京: 中国建筑工业 出版社, 2012: 3-10. [Hu Zhengfan, Lin Yulian. Environmental psychology. Beijing, China: China Architecture \& Building Press, 2012: 3-10. ]

[58] 叶浩生. 认知心理学: 困境与转向 [J]. 华东师范大学学 报 (教育科学版), 2010, 28(1): 42-47. [Ye Haosheng. Cognitive psychology: Predicament and transformation. Journal of East China Normal University (Educational Sciences Edition), 2010, 28(1): 42-47. ]

[59] Sholl M J. Landmarks, places, environments: Multiple mind-Brain systems for spatial orientation $[\mathrm{J}]$. Geoforum, 1992, 23(2): 151-164.

[60] Mealey J B, Cohen M M, Jordan K. Effects of map orientation during learning on airport identification $[\mathrm{J}]$. Aviation Space \& Environmental Medicine, 1998, 69(2): 104110.

[61] Zeisel J. Marketing therapeutic environments for Alzheimer's care $[\mathrm{J}]$. Journal of Architectural \& Planning Research, 2003, 20(1): 75-86.

[62] Hillier B. Studying cities to learn about minds: Some possible implications of space syntax for spatial cognition [J]. Environment \& Planning B, 2012, 39(1): 12-32.

[63] Tang I C, Tsai Y P, Lin Y J, et al. Using functional magnetic resonance imaging (fMRI) to analyze brain region activity when viewing landscapes $[\mathrm{J}]$. Landscape and $\mathrm{Ur}$ ban Planning, 2017, 162: 137-144.

[64] Dadvand P, Pujol J, Macià D, et al. The association between lifelong greenspace exposure and 3-dimensional brain magnetic resonance imaging in Barcelona school- 
children [J]. Environmental Health Perspectives, 2018, 126(2): 12-27.

[65] 王敏, 王盈蓄, 黄海燕, 等. 基于眼动实验方法的城市开 敞空间视觉研究: 广州花城广场案例 [J]. 热带地理, 2018, 38(6): 741-750. [Wang Min, Wang Yingxu, Huang Haiyan, et al. A visual analysis on the urban open spaces: With an eye-tracking experiment on the case study of the Flower City Square, Guangzhou. Tropical Geography, 2018, 38(6): 741-750. ]

[66] 龙瀛, 叶宇. 人本尺度城市形态: 测度、效应评估及规划 设计响应 [J]. 南方建筑, 2016(5): 41-47. [Long Ying, Ye Yu. Human-scale urban form measurements, performances, and urban planning \& design interventions. South Architecture, 2016(5): 41-47. ]

[67] Greenwald A G, Banaji M R. Implicit social cognition: Attitudes, self-esteem, and stereotypes [J]. Psychological Review, 1995, 102(1): 4-27.

[68] 朱泣, 高权. 西方地理学“情感转向”与情感地理学研究 述评 [J]. 地理研究, 2015, 34(7): 1394- 1406. [Zhu Hong, Gao Quan. Review on "emotional turn" and emotional geographies in recent western geography. Geographical Research, 2015, 34(7): 1394-1406. ]

[69] Thrift N, Dewsbury J D. Dead geographies and how to make them live again $[\mathrm{J}]$. Environment and Planning D: Society and Space, 2000, 18(4): 411-432.

[70] Davidson R J, Sutton S K. Affective neuroscience: The emergence of a discipline [J]. Current Opinion in Neurobiology, 1995, 5(2): 217-224.

[71] Lakoff G, Johnson M. Philosophy in the Flesh [M]. New York, USA: Basic Books, 1999.

[72] Johnson M, Lakoff G. Why cognitive linguistics requires embodied realism [J]. Cognitive Linguistics, 2002, 13 (3): 245-264.

[73] Connolly W E. Neuropolitics: Thinking, culture, speed [M]. Minnesota, USA: University of Minnesota Press, 2002.

[74] Lengen C, Kistemann T. Sense of place and place identity: Review of neuroscientific evidence [J]. Health \& Place, 2012, 18(5): 1162-1171.

[75] Pykett J, Enright B. Geographies of brain culture: Optimism and optimisation in workplace training programmes [J]. Cultural Geographies, 2016, 23(1): 51-68.

[76] 王敏, 林钿, 江荣影, 等. 传统节庆、身体与展演空间: 基 于人文地理学视觉量化方法的研究 [J]. 地理学报, 2017, 72(4): 671-684. [Wang Min, Lin Tian, Jiang Rong- hao, et al. Analysis of the traditional festival, body and performative space: A human geographical method from the perspective of visual quantitative research. Acta Geographica Sinica, 2017, 72(4): 671-684. ]

[77] Montag C, Diefenbach S. Towards homo digitalis: Important research issues for psychology and the neurosciences at the dawn of the internet of things and the digital society [J]. Sustainability, 2018, 10(2): 415. doi: 10.3390/ su10020415.

[78] Walls A R, Okumus F, Wang Y. Cognition and affect interplay: A framework for the tourist vacation decisionmaking process [J]. Journal of Travel \& Tourism Marketing, 2011, 28(5): 567-582.

[79] Naqvi N, Shiv B, Bechara A. The role of emotion in decision making: A cognitive neuroscience perspective [J]. Current Directions in Psychological Science, 2006, 15 (5): 260-264.

[80] Pearce P L. The experience of visiting home and familiar places [J]. Annals of Tourism Research, 2012, 39(2): 1024-1047.

[81] Goggin C L, Please P M, Ridges M J, et al. Connecting with country in Mungo national park, Australia: A case study to measure the emotional dimension of experience and place attachment $[\mathrm{J}]$. Local Environment, 2017, 22 (2): 1-20.

[82] 程国宇, 安宁, 林铭亮. 基于影视作品的情感地缘政治 研究: 以三部中国海外军事行动题材的电影为例 [J]. 世界地理研究, 2019, 28(4): 65-75. [Cheng Guoyu, An Ning, Lin Mingliang. An emotional geopolitical research on films: Taking three Chinese overseas military operations themed films as examples. World Regional Studies, 2019, 28(4): 65-75. ]

[83] Pain R. Globalized fear? Towards an emotional geopolitics [J]. Progress in Human Geography, 2009, 33(4): 466486.

[84] Ivakhiv A. From frames to resonance machines: The neuropolitics of environmental communication [J]. Environmental Communication, 2010, 4(1): 109-121.

[85] Barr S, Prillwitz J. A smarter choice? Exploring the behaviour change agenda for environmentally sustainable mobility [J]. Environment and Planning C: Government and Policy, 2014, 32(1): 1-19.

[86] Gagen E A. Governing emotions: Citizenship, neuroscience and the education of youth $[\mathrm{J}]$. Transactions of the Institute of British Geographers, 2015, 40(1): 140-152. 
[87] Bille F. Territorial phantom pains (and other cartographic anxieties) [J]. Environment and Planning D: Society and Space, 2014, 32(1): 163-178.

[88] Pykett J. Brain culture: Shaping policy through neuroscience [M]. Bristol, UK: Policy Press, 2015: 1-36.

[89] Isin E F. The neurotic citizen [J]. Citizenship Studies, 2004, 8(3): 217-235.

[90] Philo C. A 'new Foucault' with lively implications or 'the crawfish advances sideways' [J]. Transactions of the Institute of British Geographers, 2012, 37(4): 496-514.

[91] Simon H A. A behavioral model of rational choice [J]. The Quarterly Journal of Economics, 1955, 69(1): 99118.

[92] 李小建, 苗长虹. 西方经济地理学新进展及其启示 [J]. 地理学报, 2004, 59(S1): 153-161. [Li Xiaojian, Miao Changhong. Recent developments in western economic geographies and theoretical thinking for China's counterpart. Acta Geographica Sinica, 2004, 59(S1): 153-161. ]

[93] Ross D, Sharp C, Vuchinich R, et al. Midbrain mutiny: The picoeconomics and neuroeconomics of disordered gambling [M]. Cambridge, USA: MIT Press, 2008.

[94] Glimcher P W, Ernst F. Neuroeconomics: Decision making and the brain [M]. Academic Press, 2013.

[95] Basso F, Guillou L, Oullier O. Embodied entrepreneurship: A sensory theory of value $[\mathrm{M}] / /$ Stanton A A, Day M, Welpe I M. Neuroeconomics and the Firm. Cheltenham, UK: Edward Elgar Publishing, 2010: 217-232.

[96] Clark G L. Human nature, the environment, and behaviour: Explaining the scope and geographical scale of financial decision-making [J]. Geografiska Annaler: Series B, Human Geography, 2010, 92(2): 159-173.

[97] Lewis N. Myopia and the global financial crisis: Shorttermism, context- specific reasoning, and market structure [J]. Dialogues in Human Geography, 2011, 1(1): 3537.

[98] Proffitt D R. Embodied perception and the economy of action [J]. Perspectives on Psychological Science, 2006, 1 (2): 110-122.

[99] Oullier O, Sauneron S. Improving public health prevention with behavioural, cognitive and neuroscience [M]. Paris, France: Centre d'Analyse Stratégique, 2010.

[100] Grasso M. Climate ethics: With a little help from moral cognitive neuroscience $[\mathrm{J}]$. Environmental Politics, 2013, 22(3): 377-393.

[101] Yalachkov Y, Naumer M J, Plyushteva A. The compul- sive habit of cars $[\mathrm{J}]$. Trends in Cognitive Sciences, 2014, 18(5): 227-8. doi: 10.1016/j.tics.2014.01.008.

[102] Jones R, Pykett J, Whitehead M. Changing behaviours: On the rise of the psychological state [M]. Cheltenham, UK: Edward Elgar Publishing, 2013.

[103] Berndt C. Behavioural economics, experimentalism and the marketization of development $[\mathrm{J}]$. Economy and Society, 2015, 44(4): 567-591.

[104] 陈巍. 认知神经科学技术在心理学上的应用 [J]. 心理 技术与应用, 2013(2): 32-34. [Chen Wei. The application of cognitive neuroscience techniques in psychology. Psychology: Techniques and Applications, 2013(2): 32-34. ]

[105] 刘宝根, 周貺, 李菲菲. 脑功能成像的新方法: 功能性 近红外光谱技术(fNIRS) [J]. 心理科学, 2011(4): 943949. [Liu Baogen, Zhou Jing, Li Feifei. Functional Near- Infrared Spectroscopy: An Emerging Functional Neuroimaging Technology. Journal of Psychological Science, 2011(4): 943-949. ]

[106] Roberts A C, Christopoulos G I, Car J, et al. Psycho-biological factors associated with underground spaces: What can the new era of cognitive neuroscience offer to their study? [J]. Tunnelling and Underground Space Technology, 2016, 55(1): 118-134.

[107] Brett M, Johnsrude I S, Owen A M. The problem of functional localization in the human brain $[\mathrm{J}]$. Nature Reviews Neuroscience, 2002, 3(3): 243-249.

[108] 张泽宝. 医学影像物理学 [M]. 北京: 人民卫生出版 社, 2000. [Zhang Zebao. Medical imaging physics. Beijing, China: People's Medical Publishing House, 2000. ]

[109] Lederbogen F, Kirsch P, Haddad L, et al. City living and urban upbringing affect neural social stress processing in humans [J]. Nature, 2011, 474: 498-501.

[110] 王敏, 江荣影, 朱泣. 人文地理学的“视觉”研究进展与 启示 [J]. 人文地理, 2017, 32(3): 10-19. [Wang Min, Jiang Ronghao, Zhu Hong. A review and revelation of the study of visual in human geography. Human Geography, 2017, 32(3): 10-19. ]

[111] 李奇, 耿海燕. 视觉意识的认知神经科学研究进展 [J]. 自然科学进展, 2008, 18(11): 1211-1219. [Li Qi, Geng Haiyan. Advances in the cognitive neuroscience of visual awareness. Progress in Natural Science, 2008, 18 (11): 1211-1219. ]

[112] 董卫华, 廖华, 詹智成, 等. 2008 年以来地图学眼动与 视觉认知研究新进展 [J]. 地理学报, 2019, 74(3): 599- 
614. [Dong Weihua, Liao Hua, Zhan Zhicheng, et al. New research progress of eye tracking-based map cognition in cartography since 2008. Acta Geographica Sinica, 2019, 74(3): 599-614. ]

[113] 李伟, 陈毓芬. 地图学眼动研究及实验参数解析 [J]. 测 绘通报, 2012(10): 16-20. [Li Wei, Chen Yufen. Cartography eye movements study and the experimental parameters analysis. Bulletin of Surveying and Mapping, 2012(10): 16-20. ]

[114] 王君怡, 林岗, 高华, 等. 大学生旅游地图空间符号认 知的群体差异研究: 基于眼动实验数据分析 [J]. 旅游 学刊, 2016, 31(3): 97-105. [Wang Junyi, Lin Lan, Gao Hua, et al. Differences in college students' spatial symbol cognition of tourism map: Based on experimental data from an eye-movement tracking system. Tourism Tribune, 2016, 31(3): 97-105. ]

[115] Wang Y, Sparks B A. An eye-tracking study of tourism photo stimuli image characteristics and ethnicity [J]. Journal of Travel Research, 2016, 55(5): 588-602.

[116] 王敏, 江冰婷, 朱竑. 基于视觉研究方法的工业遗产旅 游地空间感知探讨: 广州红专厂案例 [J]. 旅游学刊, 2017, 32(10): 28-38. [Wang Min, Jiang Bingting, Zhu Hong. The spatial perception of tourism destination based on visual methodology: The case study of Guangzhou redtory. Tourism Tribune, 2017, 32(10): 28-38. ]

[117] Wang M, Zhao M, Lin M, et al. Seeking lost memories: Application of a new visual methodology for heritage protection [J]. Geographical Review, 2020. doi: 10.1080/ 00167428.2020.1715800.
[118] Bodor P, Illes A. Possibilities of analyzing visual conduct with an eyetracker device: searching for visual dialects [J]. Poznań Studies in Contemporary Linguistics, 2008, 44(2): 197-213.

[119] Wulff- Jensen A. Data conversion tool for Tobii pro glasses 2 live data files: From .json to .txt [R/OL]. Technical Report, 2016. doi: 10.13140/RG.2.2.26895.28321.

[120] Knez I, Niedenthal S. Lighting in digital game worlds: Effects on affect and play performance [J]. Cyber Psychology \& Behavior, 2008, 11(2): 129-137.

[121] 周尚意, 苏娴. 面向城市基调管控的建筑群色彩感知 和意义理解分析: 以北京故宫建筑群为例 [J]. 人文地 理, 2019, 34(4): 32-39. [Zhou Shangyi, Su Xian. The color perception and color meaning awareness analysis of architectures for urban context planning: A case of the forbidden city in Beijing. Human Geography, 2019, 34(4): 32-39. ]

[122] Roepstorff A, Frith C. Neuroanthropology or simply anthropology? Going experimental as method, as object of study, and as research aesthetic [J]. Anthropological Theory, 2012, 12(1): 101-111.

[123] Von Scheve C. Sociology of neuroscience or neurosociology? [M]// Rothman B K. Sociological reflections on the neurosciences. Bingley, UK: Emerald Group Publishing, 2011: 255-278.

[124] Roberts A C, Christopoulos G I. Comment on "Using functional Magnetic Resonance Imaging (fMRI) to analyze brain region activity when viewing landscapes" [J]. Landscape and Urban Planning, 2018, 172: 25-28. 


\title{
Research progress and implication of the "neural turn" in human geography
}

\author{
WANG Min ${ }^{1}$, LIN Mingliang ${ }^{2}$, ZHU Hong ${ }^{3 *}$ \\ (1. School of Geography, South China Normal University, Guangzhou 510631, China; \\ 2. School of Urban and Regional Science, East China Normal University, Shanghai 200241, China; \\ 3. Centre for Human Geography and Urban Development, Guangzhou University, Guangzhou 510006, China)
}

\begin{abstract}
Neuroscience is the science of the 21st century. The theoretical research results of neuroscience are affecting daily life, environment, space, context, and place profoundly. It provides a new way of thinking and method to explain human's mind and behavior. In the context of post-modernism, the understanding of selfconsciousness in human geography has become fragmented. Non-presentation theory and embodied cognitive geography claim that in most of the time, people are "unconscious". Subject self-consciousness is irrational, which takes place in the "outflows" daily life world. It emphasizes the showing, performance, and practice of the body. The meaning of the real world should be obtained through embodied cognition and behavior. They found evidence from neuroscience that led to a "neural turn" in geography. The "neural turn" devotes to linking human's mind and behavioral traits to biological evidence. It is different from inter-disciplinary debates that are breaking down the disciplinary boundaries between social culture and natural sciences. The laboratory methods cannot reflect the real world, and the social science methods are difficult to get rid of subjective factors. The "neural turn" can provide a research perspective of combining the-first-person and the-third-person for human geography. This article reviewed the recent studies on the "neural turn" in Western human geography. "Neural turn" research mainly concentrated in three aspects: space perception, embodied affect, and behavior practice. It has involved economic, political, social, cultural, public policy, tourism, and education topics. The application of neuroscience in human geography can also be applied to multiple scales of space such as country, city, community, and body. Neuroscience perspective as a new path is used in urban planning, environmental design, tourism development, and public policy, among others. At the same time, the technical tools of neuroscience have become new data collection methods in human geography. Neural technology can capture imperceptible physiological changes in the human body in order to detect neural activity caused by mind and behavior in a particular environment and context. The collection of physiological data can surmount the representation of text and symbols to discover non- representational results. Brain images and eye tracking technologies have been applied in geography, presenting predictable visions for the future. At present, Chinese human geography pays little attention to neuroscience. This article is hoped to provide some reference and enlightenment for the study of Chinese human geography. The dialogue between neuroscience and human geography is still in its infancy and needs more empirical research. In this process, human geography can carry out more cooperative research to produce more knowledge spillover. At the same time, these studies need to explore the topic of Chinese localization in order to better realize the people-oriented approach in the research and application of Chinese human geography.
\end{abstract}

Keywords: neuroscience; non-representational theories; embodied cognition; mind; behavior; human geography 